\title{
Identification of definitive serum biomarkers associated with disease activity in primary Sjögren's syndrome
}

\author{
Ayumi Nishikawa' ${ }^{1}$ Katsuya Suzuki ${ }^{1}$, Yoshiaki Kassai ${ }^{2}$, Yuumi Gotou², Maiko Takiguchi², Takahiro Miyazaki ${ }^{2}$, \\ Keiko Yoshimoto', Hidekata Yasuoka', Kunihiro Yamaoka', Rimpei Morita ${ }^{3}$, Akihiko Yoshimura $^{3}$ \\ and Tsutomu Takeuchi ${ }^{1^{*}}$
}

\begin{abstract}
Background: In this study, we sought to identify definitive biomarkers associated with disease activity in primary Sjögren's syndrome (pSS).

Methods: Serum protein concentrations in pSS patients and healthy controls (HCs) were comprehensively screened using high-throughput proteomic analysis, and differentially expressed proteins were extracted. Correlation between differentially expressed proteins and European League Against Rheumatism Sjögren's Syndrome Disease Activity Index (ESSDAI) scores was analyzed and disease activity-associated biomarkers were identified. These biomarkers were validated by enzyme-linked immunosorbent assay (ELISA) in a separate pSS cohort.

Results: The serum concentrations of 1100 proteins were compared between 30 pSS patients and 30 HCs, with 82 differentially expressed proteins identified as pSS-associated proteins. Of these 82 proteins, 9 were identified as disease activity-associated biomarkers. These nine biomarkers underwent validation by ELISA in a separate pSS validation cohort ( $n=58$ ), with five proteins (CXCL13, TNF-R2, CD48, B-cell activating factor (BAFF), and PD-L2) subsequently being confirmed as candidate biomarkers. Of these five candidate biomarkers, CXCL13 exhibited the most significant correlation with the lymphadenopathy, glandular, and pulmonary domains of the ESSDAI. CXCL13, TNF-R2 and CD48 exhibited a positive correlation with the biological domain of the ESSDAI. TNF-R2 exhibited the most negative correlation with uptake in the submandibular gland on technetium 99m-pertechnetate salivary gland scintigraphy.
\end{abstract}

Conclusions: Our approach successfully identified serum biomarkers associated with disease activity in pSS patients. These markers might be potential therapeutic targets in pSS patients.

Keywords: Sjögren's syndrome, Biomarker, Proteomics, Serum protein, Disease activity

\section{Background}

Primary Sjögren's syndrome (pSS) is a systemic autoimmune disease characterized by dry eyes and dry mouth, and by systemic manifestations, such as general fatigue and fever, and damage to multiple organs [1]. Immunological abnormalities such as antinuclear antibodies (ANAs), antibodies to SS-A or SS-B, and hypergammaglobulinemia are often detected in PSS patients by laboratory tests [2,3]. Infiltration of lymphocytes in salivary or

\footnotetext{
* Correspondence: tsutake@z5.keio.jp

'Division of Rheumatology, Department of Internal Medicine, Keio University School of Medicine, 35 Shinanomachi, Shinjuku-ku, Tokyo 160-8582, Japan Full list of author information is available at the end of the article
}

lachrymal glands is typically observed in affected patients, which results in destruction and subsequent fibrotic changes [3-6]. However, the pathogenesis of pSS remains unclear due to the heterogeneity of clinical phenotypes and complex pathogenetic mechanisms. The identification of disease-associated molecular clusters or biomarkers will therefore help to clarify the complex pathogenesis of pSS.

Previous studies attempted to identify novel biomarkers that reflect pSS pathogenesis, using traditional proteomic approaches such as two-dimensional electrophoresis or mass spectrometry to characterize protein expression profiles in lachrymal or salivary fluid [7-14]. Most of these profiles consist of secretory proteins, 
enzymes, and highly abundant immune-related proteins such as albumin and $\beta 2$-microglobulin ( $\beta 2 M G$ ). However, given that the roles of these biomarkers in pathogenesis are unclear, they are not used at the clinical level.

B-cell-activating factor (BAFF), $\beta 2 \mathrm{MG}$ and myxovirus resistance protein $\mathrm{A}(\mathrm{MxA})$ were recently identified as biomarkers that correlate with European League Against Rheumatism (EULAR) Sjögren's Syndrome Disease Activity Index (ESSDAI) scores [15-17], which is an objective method of evaluating clinical disease activity in clinical pSS research $[1,4,18,19]$. BAFF belongs to the tumor necrosis factor family and levels are slightly higher in the serum of pSS patients with lymphoproliferative disorders or clonal B-cell expansion in the salivary glands than pSS patients without these disorders. Serum $32 \mathrm{MG}$ is significantly higher in patients with pSS with history of lymphoma than in the others. MxA is a key mediator of the interferon (IFN)-induced antiviral response and is tightly regulated by type I IFNs. MxA is associated with a systemic type I IFN signature in certain subsets of patients with pSS. These studies demonstrate the clinical significance of the three biomarkers associated with the ESSDAI.

Here, we extracted disease-related molecular clusters and definitive protein biomarkers associated with pSS disease activity as assessed by the ESSDAI score, utilizing a novel comprehensive high-throughput proteomics analysis of more than 1100 proteins. We also validated the candidate biomarkers by ELISA in a separate pSS validation cohort.

\section{Methods}

\section{Patients and controls}

A total of 88 patients with primary Sjögren's syndrome (pSS) meeting at least one of the following criteria: the 2002 American-European criteria for SS (AECG) [20]; the 2012 American College of Rheumatology (ACR) classification criteria for pSS [21]; or the revised Japanese Ministry of Health criteria for the diagnosis of SS [22], who had provided written informed consent and were returning for follow up at Keio University Hospital, were enrolled from April 2011 to July 2014. Of these 88 patients, 30 were analyzed in the initial cohort and the remaining 58 in the validation cohort; 40 of 88 patients satisfied the AECG criteria, 61 satisfied the ACR criteria, and 54 satisfied the Japanese criteria.

Patients who were being treated with moderate to high doses of corticosteroids, immunosuppressants, or biological agents were excluded. Thirty healthy individuals who did not suffer from autoimmune diseases or were not receiving any drugs were included as controls. Information on patient demographics and clinical parameters were retrospectively collected from medical records. All procedures were approved by the medical ethics committee of Keio University Hospital and followed the tenets of the Declaration of Helsinki. All samples and information were collected after patients and controls gave written informed consent.

\section{Clinical and histological assessments}

Disease activity in pSS was quantified based on the ESSDAI score. The ESSDAI score evaluates 12 domains. Each domain is divided into three to four levels according to the degree of activity and scored as 0 (no activity), 1 (low activity), 2 (moderate activity) or 3 (high activity) [23]. The following tests were used to objectively assess the dryness of the eyes: Schirmer's test, Rose Bengal (RB) score test, and fluorescein clearance test. The gum test was used as an indicator of oral dryness, and technetium $99 \mathrm{~m}$-pertechnetate scintigraphy was used to assess salivary gland function using standard clinical methods. Histological analysis was conducted using hematoxylin-eosin staining of lip biopsy specimens.

\section{Serum isolation and storage}

After blood samples were collected from donors in tubes with serum-separating agent, serum was immediately separated by centrifugation, and several aliquots were stored at $-80^{\circ} \mathrm{C}$ until use.

\section{Comprehensive high-throughput screening of serum protein concentrations}

Serum protein concentration was measured using a Slow Off-rate Modified DNA Aptamer (SOMAmer)-based capture array (SOMAscan ${ }^{\text {TM}}$; SomaLogic, Inc., Boulder, CO, USA), which is a comprehensive high-throughput proteomics assay using an Agilent microarray readout that measures 1128 proteins [24, 25]. Briefly, $75 \mu \mathrm{l}$ of serum from each sample was incubated with a mixture of the 1128 SOMAmer $^{\circ}$ reagents that specifically bind to each protein and was incubated in separate wells on a 96-well plate. Each protein SOMAmer complex was then biotinylated and captured by streptavidin beads. SOMAmer was then removed and measured in relative fluorescence units (RFU) based on the fluorescent SOMAmer hybridized to a complementary probe on custom microarray slides [26], and the level of RFU was then converted to serum protein concentration.

\section{Enzyme-linked immunosorbent assay (ELISA)}

Disease activity-associated biomarkers positively that were correlated with the ESSDAI scores were applied to validation analysis in different cohorts using ELISA. Briefly, serum samples were separated and stored at $-80{ }^{\circ} \mathrm{C}$ until analysis. After thawing, the assay was performed in accordance with the manufacturer's instructions. Concentrations of those biomarkers were 
measured and quantified using a spectrophotometer (iMark Microplate Absorbance Reader, Bio Rad, CA, USA).

The following commercially available kits for ELISA were used: Human Ephrin Type-B Receptor 2 (EPHB2) ELISA Kit (CUSABIO, Wuhan, Hubei Province P.R., China), Quantikine Human CXCL13/BLC/BCA-1 Immunoassay ELISA kit (R\&D Systems, Minneapolis, MN, USA), Human CD48 ELISA Pair Set (Sino Biological Inc., Beijing P.R., China), $\beta-2$ Microgloblin Human SimpleStep ELISA ${ }^{\mathrm{TM}}$ Kit, (Abcam, Tokyo, Japan), Soluble TNF Receptor II Human ELISA Kit (Abcam), RayBio ${ }^{\circ}$ Human LAG3 ELISA Kit (RayBiotech, Inc., Norcross, GA, USA), DuoSet ${ }^{\circ}$ ELISA Human CD163 and DuoSet ${ }^{\circ}$ Ancillary Reagent Kit2 (R\&D Systems), and ELISA Kit for Programmed Cell Death Protein 1 Ligand 2 (PDCD1LG2) (Cloud-Clone Corp., Houston, TX, USA). For detection of BAFF, human BAFF affinity-purified polyclonal antibody and human BAFF biotinylated affinity-purified polyclonal antibody (R\&D Systems) were used as previously described [27].

\section{Algorithm for identifying disease-related molecular clusters and definitive serum protein biomarkers associated with disease activity}

Figure 1 shows the strategy for identifying diseaserelated molecular clusters and novel serum protein biomarkers associated with disease activity. A total of 1128 serum proteins in $30 \mathrm{pSS}$ patients and 30 healthy controls (HCs) were comprehensively screened using SOMAscan ${ }^{\text {TM }}$. Twenty-eight serum proteins were excluded due to lack of acuity in measurement. Mean concentrations of the remaining 1100 proteins in the pSS and $\mathrm{HC}$ groups were compared. Differentially expressed serum proteins were selected as pSS-associated proteins based on the following criteria: $P<0.05$ for comparison of protein concentrations in patients with pSS and HCs using the Mann-Whitney $U$ test, and fold-change in trimmed mean protein concentrations $>1.2$ or $<0.83$ in patients with pSS compared to HCs.

In enrichment analysis, biological interpretation of pSSassociated proteins was performed with original analysis software MoCo3 (Takeda Pharmaceutical Co., Ltd., Tokyo, Japan). First, the names of pSS-associated proteins were converted into gene symbols in public databases (further information available at http://www.ncbi.nlm.nih.gov/biosystems, http://genome.ucsc.edu/, http://www.ncbi.nlm.nih.gov/gap, http://www.genome.gov/gwastudies/, http:// www.ncbi.nlm.nih.gov/, http://thebiogrid.org/, and http:// hugenavigator.net/HuGENavigator/home.do) and a commercial database (BIOBASE Knowledge Library). MoCo3 evaluates the overlap between gene sets using Fisher's exact test (a $P$ value $<0.01$ is considered significant) and visualizes the statistical significant associations between them in a graph (network). The statistical methods and other computational algorithms of $\mathrm{MoCo} 3$ were as previously described [28].

For analysis of correlation between ESSDAI and serum protein concentration for continuous values, Spearman's rho test (a $P$ value $<0.01$ was considered significant) was used for analysis in the pSS initial cohort $(n=30)$ examined by SOMAscan ${ }^{\mathrm{TM}}$, and Pearson's correlation coefficient test (a $P$ value $<0.05$ was considered significant) was used for the separate pSS validation cohort $(n=58)$ examined by ELISA. Finally, disease activity-associated biomarkers were compared with clinical variables, including clinical laboratory tests, clinical examinations, and imaging tests for salivary gland function using Pearson's correlation coefficient test (a $P$ value $<0.05$ was considered significant). All analyses were conducted using $\mathrm{JMP}^{\circ}$ software, version 11.0 (SAS Institute Inc., Cary, NC, USA).

\section{Results}

Extraction of differentially expressed serum proteins in patients with pSS

Clinical characteristics of patients and controls are shown in Table 1 . The ratio of women to men was almost equal in both patients with pSS and HCs. Mean age of patients with pSS was $61.1 \pm 10.8$ years, which was higher than that of HCs. Systemic activity in pSS was low overall, and the mean ESSDAI score was 2.6. Only one patient required treatment with corticosteroids during evaluation.

In total 82 serum proteins that were differentially expressed in patients with pSS and HCs were extracted as pSS-associated proteins from 1128 proteins, based on a combination of statistical differences in serum concentration and fold-change in serum concentration. A total of 57 upregulated and 25 downregulated proteins were identified, along with the fold-change versus mean value of HCs, and the $P$ value was calculated using the MannWhitney $U$ test (Additional file 1: Table S1).

\section{Characteristics of disease-related molecular clusters in patients with pSS}

To identify molecular clusters in the 82 pSSassociated proteins, enrichment analysis was applied as shown in Additional file 2: Figure S1. Characteristics of the serum protein signature in patients with pSS included the following molecular concepts: "extracellular region", "chemokine signaling pathway", "downstream of TNF- $\alpha$ ", "platelet activation", and "platelet degranulation". These molecular concepts were classified into immune response-related and platelet-related molecular clusters. 


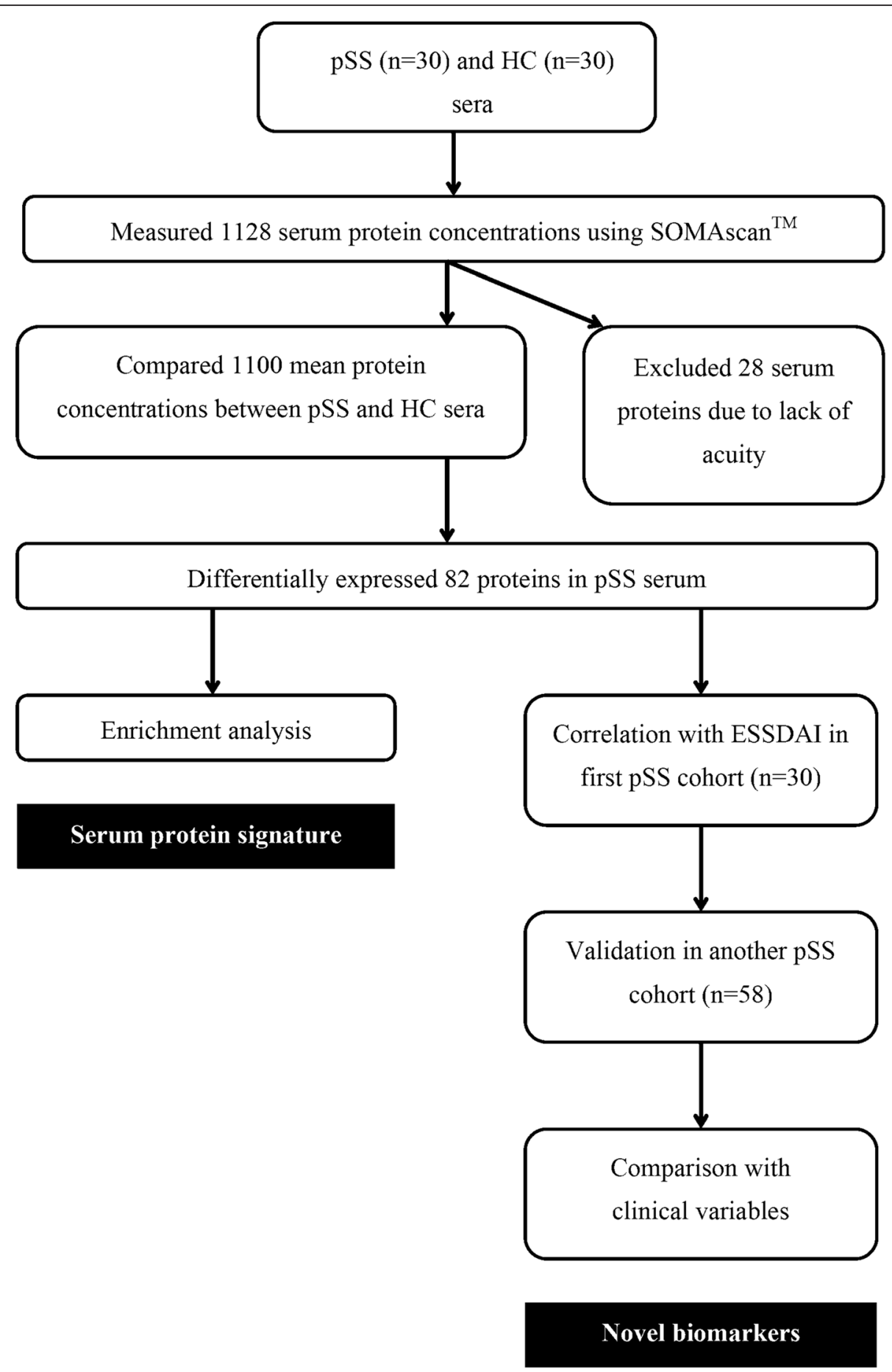

Fig. 1 Overview of strategy for identification of serum proteins associated with disease activity and protein signature in patients with primary Sjögren's syndrome (pSS). HC healthy controls, ESSDAl European League Against Rheumatism Sjögren's Syndrome Disease Activity Index

\section{Screening of proteins correlated with clinical disease} activity in pSS

To extract disease activity-associated biomarkers, the correlation between serum protein levels and ESSDAI scores in patients with pSS was tested. Nine proteins were statistically extracted by Spearman's rho test (Table 2) as follows: ephrin type-B receptor 2 (EPHB-2), C-X-C motif chemokine 13 (CXCL13), signaling lymphocytic activation molecule 2 (SLAMF-2, CD48), $32 \mathrm{MG}$, BAFF, TNF receptor 2 (TNF-R2), lymphocyte activation gene-3 (LAG-3), cluster of differentiation 163 (CD163), and programmed cell death protein 1 ligand 2 (PD-L2). 
Table 1 Characteristics of patients and controls

\begin{tabular}{lll}
\hline Characteristic & $\begin{array}{l}\text { Healthy controls } \\
(n=30)\end{array}$ & $\begin{array}{l}\text { Patients with pSS } \\
(n=30)\end{array}$ \\
\hline $\begin{array}{l}\text { Demographic } \\
\text { Female, n (\%) }\end{array}$ & $30(100)$ & $29(97)$ \\
Age, mean (SD), years ${ }^{*}$ & $39.9(9.4)$ & $61.1(10.8)$ \\
$\begin{array}{l}\text { Disease duration, mean (SD), } \\
\text { years }\end{array}$ & NA & $4.7(6.6)$ \\
Clinical manifestation, $n$ (\%) & & \\
Ocular symptoms & NA & $18(60)$ \\
Oral symptoms & NA & $21(70)$ \\
Objectively assessed dryness & NA & $24(80)$ \\
Anti-SSA positivity & NA & $24(80)$ \\
Anti-SSB positivity & NA & $14(48)$ \\
Lymphocytic sialadenitis with & NA & $12(40)$ \\
focus score $\geq 1$ & & $2.6(4.2)$ \\
ESSDAl, mean (SD) & NA & \\
Treatment & & $1(3)$ \\
Corticosteroid & NA & $0(0)$ \\
Immunosuppressant & NA
\end{tabular}

"Data are presented as mean \pm standard deviation (SD) or number (\%). pSS primary Sjögren's syndrome, ESSDAl the European League Against Rheumatism Sjögren's Syndrome Disease Activity Index, NA not applicable

\section{Identification of novel disease activity-associated biomarkers positively correlated with ESSDAI scores in the pSS validation cohort}

To confirm that the association between disease activityassociated biomarkers and ESSDAI scores was reproducible, a validation cohort consisting of serum samples from another 58 patients with pSS was analyzed. No marked differences in background characteristics were noted between the initial and validation cohorts (Table 3).

Table 2 Serum proteins positively correlated with ESSDAI score in patients with pSS in first cohort $(n=30)$

\begin{tabular}{lcl}
\hline Protein & Spearman's $\rho$ & $P$ value \\
\hline EPHB-2 & 0.65 & $<0.0001$ \\
CXCL13 & 0.60 & 0.0005 \\
CD48 & 0.56 & 0.0014 \\
$\beta 2 M G$ & 0.55 & 0.0018 \\
BAFF & 0.52 & 0.0029 \\
TNF-R2 & 0.51 & 0.0039 \\
LAG-3 & 0.49 & 0.0058 \\
CD163 & 0.49 & 0.0062 \\
PD-L2 & 0.47 & 0.0081
\end{tabular}

Spearman's rho correlation coefficient test was used to test correlation between 82 differentially expressed protein concentrations and the European League Against Rheumatism Sjögren's Syndrome Disease Activity Index (ESSDAI) scores. $E P H B-2$, ephrin type-B receptor $2, C X C L 13, C-X-C$ motif chemokine $13, \beta 2 M G \beta 2$ microglobulin, BAFF B-cell activating factor, TNF-R2 TNF receptor 2, LAG-3, lymphocyte activation gene-3, PD-L2 programmed cell death protein 1 ligand $2 A$ $P$ value $<0.01$ was considered significant
Serum concentrations of nine candidates were measured using an ELISA and their correlation with the ESSDAI was statistically analyzed (Fig. 2). There was significant correlation between ESSDAI scores and CXCL13, TNFR2, CD48, BAFF, and PD-L2 in both the initial and the validation cohorts of patients with $\mathrm{pSS}$.

\section{Association between disease activity-associated biomarkers and clinical characteristics of patients with pSS}

To characterize serum biomarkers of clinical significance, the correlation between the five disease activityassociated biomarkers and various clinical parameters in the pSS validation cohort $(n=58)$ was assessed (Table 4$)$. Notably, these five biomarkers were positively correlated with three domains of the ESSDAI, the lymphadenopathy, glandular, and pulmonary domains. In our pSS patient cohort, CXCL13 correlated significantly with the ESSDAI score and these three domains of the ESSDAI, and the strength of the correlation between the ESSDAI and TNF-R2, CD48, BAFF, and PD-L2, respectively, continued in this order. In addition, the serum concentrations of CXCL13, TNF-R2, and CD48 were positively correlated with that of immunoglobulin (Ig) $\mathrm{G}$ and the biological domain of the ESSDAI score.

The associations were further investigated between these biomarkers, and clinical examinations, imaging tests for salivary gland function, and histological grade. TNF-R2 was negatively correlated with unstimulated salivary flow as assessed by the Gum test. CXCL13, TNF$\mathrm{R} 2$, and BAFF were negatively correlated with uptake in the submandibular gland on technetium 99mpertechnetate salivary gland scintigraphy, with TNF-R2 exhibiting the strongest correlation. In addition, only BAFF was negatively correlated with the excretion rate in the submandibular gland.

\section{Discussion}

We conducted a comprehensive study of serum proteins in patients with pSS using the most recent and reliable high-throughput proteomics approach, with simultaneous screening of more than 1100 multiple proteins. We identified pSS-associated molecular clusters and validated disease activity-associated biomarkers in a larger cohort than in previous studies of biomarkers for pSS [7-15, 17]. We also analyzed the association between disease activityassociated biomarkers and clinical characteristics.

We first conducted enrichment analysis to clarify the presence of a distinct serum protein signature of pSS and found that the majority of pSS-associated proteins were involved in the immune response-related or the plateletrelated molecular cluster. The immune response-related molecular cluster indicates altered immune responses, such as upregulated chemokine or cytokine expression and chemotaxis activation. This in turn suggests that an 
Table 3 Characteristics of patients in the validation and initial cohorts

\begin{tabular}{lll}
\hline Characteristic & Validation cohort $(n=58)$ & Initial cohort $(n=30)$ \\
\hline Demographic & & $29(97)$ \\
Female, $n(\%)$ & $60.3(13.3)$ & $61.1(10.8)$ \\
Age, mean (SD), years ${ }^{*}$ & $3.5(5.8)$ & $4.7(6.6)$ \\
Disease duration, mean (SD), years ${ }^{*}$ & & $18(60)$ \\
Clinical manifestation, $n$ (\%) & $39(67)$ & $21(70)$ \\
Ocular symptoms & $53(91)$ & $24(80)$ \\
Oral symptoms & $48(83)$ & $24(80)$ \\
Objectively assessed dryness & $43(74)$ & $14(48)$ \\
Anti-SSA positivity & $24(41)$ & $12(40)$ \\
Anti-SSB positivity & $10(17)$ & $2.6(4.2)$ \\
Lymphocytic sialadenitis with focus score $\geq 1_{\text {ESSDAl, mean score (SD) }}^{*}$ & $2.6(4.9)$ & $1(3)$ \\
Treatment & & $0(0)$
\end{tabular}

*Data are presented as mean \pm SD or number (\%). Anti-SSA anti-Sjögren's syndrome antigen A antibodies, Anti-SSB anti-Sjögren's syndrome antigen B antibodies, ESSDAI European League Against Rheumatism Sjögren's Syndrome Disease Activity Index

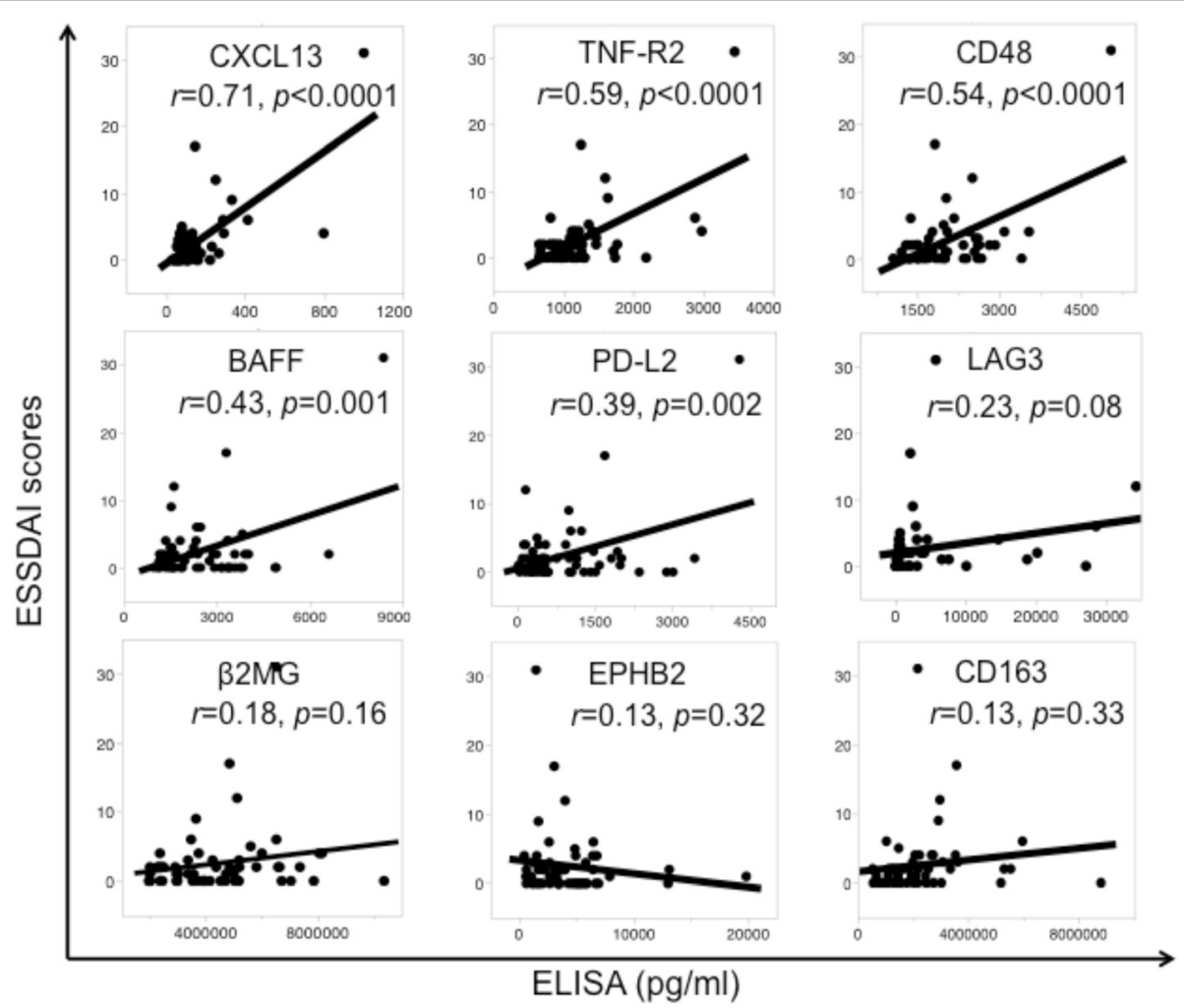

Fig. 2 Correlation between European League Against Rheumatism Sjögren's Syndrome Disease Activity Index (ESSDA) scores and concentrations of nine screened serum proteins analyzed by ELISA in the validation cohort of patients with primary Sjögren's syndrome $(n=58)$. The nine proteins were CXCL13, TNF receptor 2 (TNF-R2), CD48, B-cell activating factor (BAFF), programmed cell death protein 1 ligand 2 (PD-L2), lymphocyte activation gene-3 (LAG-3), $\beta 2$-microglobulin (B2MG), ephrin type-B receptor 2 (EPHB2), and CD163. Pearson's correlation test was used. The correlation coefficients ( $r$ ) and $P$ values are shown in the table. $P<0.05$ was considered significant 
Table 4 Clinical variables associated with five disease activity-associated biomarkers

\begin{tabular}{|c|c|c|c|c|c|c|c|c|c|c|c|}
\hline \multirow[t]{2}{*}{ Variable } & \multirow[t]{2}{*}{$\mathrm{N}$} & \multicolumn{2}{|l|}{ CXCL13 } & \multicolumn{2}{|c|}{ TNF-R2 } & \multicolumn{2}{|l|}{ CD48 } & \multicolumn{2}{|l|}{ BAFF } & \multicolumn{2}{|l|}{ PD-L2 } \\
\hline & & $r$ & $P$ & $r$ & $P$ & $r$ & $P$ & $r$ & $P$ & $r$ & $P$ \\
\hline ESSDAl score & 58 & 0.7 & $<0.0001$ & 0.59 & $<0.0001$ & 0.54 & $<0.0001$ & 0.43 & 0.001 & 0.39 & 0.002 \\
\hline Constitutional & & NA & NA & NA & NA & NA & NA & NA & NA & NA & NA \\
\hline Lymphadenopathy & & 0.69 & $<0.0001$ & 0.54 & $<0.0001$ & 0.58 & $<0.0001$ & 0.58 & $<0.0001$ & 0.5 & $<0.0001$ \\
\hline Glandular & & 0.44 & 0.001 & 0.37 & 0.004 & 0.33 & 0.01 & 0.43 & 0.001 & 0.3 & 0.02 \\
\hline Articular & & 0.06 & 0.62 & 0.14 & 0.3 & -0.03 & 0.8 & 0.11 & 0.39 & -0.08 & 0.57 \\
\hline Cutaneous & & NA & NA & NA & NA & NA & NA & NA & NA & NA & NA \\
\hline Pulmonary & & 0.65 & $<0.0001$ & 0.52 & $<0.0001$ & 0.52 & $<0.0001$ & 0.38 & 0.003 & 0.34 & 0.01 \\
\hline Renal & & NA & NA & NA & NA & NA & NA & NA & NA & NA & NA \\
\hline Muscular & & NA & NA & NA & NA & NA & NA & NA & NA & NA & NA \\
\hline Peripheral nervous system & & NA & NA & NA & NA & NA & NA & NA & NA & NA & NA \\
\hline Central nervous system & & -0.001 & 0.1 & 0.01 & 0.94 & -0.04 & 0.79 & 0.07 & 0.59 & 0.1 & 0.41 \\
\hline Hematological & & 0.17 & 0.2 & 0.1 & 0.47 & 0.02 & 0.9 & -0.17 & 0.21 & -0.13 & 0.32 \\
\hline Biological & & 0.5 & $<0.0001$ & 0.42 & 0.001 & 0.45 & 0.0004 & 0.11 & 0.4 & 0.2 & 0.13 \\
\hline $\lg G$ & 55 & 0.62 & $<0.0001$ & 0.5 & $<0.0001$ & 0.57 & $<0.0001$ & 0.16 & 0.25 & 0.12 & 0.38 \\
\hline C3 & 52 & -0.12 & 0.39 & -0.06 & 0.69 & -0.24 & 0.09 & 0.04 & 0.77 & 0.01 & 0.96 \\
\hline C4 & 52 & 0.13 & 0.37 & 0.24 & 0.08 & 0.18 & 0.2 & 0.2 & 0.15 & 0.13 & 0.37 \\
\hline Schirmer test ( $\mathrm{mm} / 5 \mathrm{~min})$ & 21 & 0.03 & 0.9 & -0.28 & 0.23 & -0.12 & 0.6 & -0.38 & 0.09 & 0.23 & 0.32 \\
\hline RB test (score) & 14 & -0.04 & 0.9 & 0.18 & 0.55 & 0.17 & 0.57 & 0.05 & 0.86 & -0.16 & 0.58 \\
\hline Fluorescein (score) & 17 & 0.19 & 0.45 & 0.23 & 0.38 & -0.12 & 0.65 & 0.3 & 0.23 & 0.2 & 0.44 \\
\hline Gum test (ml) & 28 & -0.18 & 0.35 & -0.4 & 0.03 & -0.28 & 0.15 & -0.2 & 0.3 & -0.02 & 0.91 \\
\hline GS grade (score) & 23 & 0.08 & 0.72 & 0.13 & 0.54 & 0.23 & 0.29 & 0.28 & 0.2 & 0.23 & 0.3 \\
\hline Scintigraphy & 40 & & & & & & & & & & \\
\hline PG uptake ratio (\%) & & -0.18 & 0.26 & -0.27 & 0.1 & -0.01 & 0.95 & 0.01 & 0.94 & -0.13 & 0.44 \\
\hline SMG uptake ratio (\%) & & -0.48 & 0.004 & -0.52 & 0.001 & -0.28 & 0.1 & -0.43 & 0.01 & -0.15 & 0.4 \\
\hline PG excretion ratio (\%) & & 0.12 & 0.45 & 0.06 & 0.7 & 0.02 & 0.9 & -0.05 & 0.77 & -0.08 & 0.64 \\
\hline SMG excretion ratio (\%) & & 0.05 & 0.79 & 0.26 & 0.18 & 0.09 & 0.62 & -0.49 & 0.01 & 0.26 & 0.17 \\
\hline
\end{tabular}

Candidate biomarkers and clinical variables are shown in the header row and left columns, respectively. Data are presented as Pearson's correlation coefficients ( $r$ ) and $P$ values $(P)$. A $P$ value $<0.05$ was considered significant. TNFR TNF receptor, BAFF B-cell activating factor, PD-L2 programmed cell death protein 1 ligand 2 , ESSDAl score the European League Against Rheumatism Sjögren's Syndrome Disease Activity Index (ESSDAl) score, Schirmer's test Schirmer's / test (mm/5 min), RB test Rose Bengal staining (score: van Bijsterveld score), Fluorescein fluorescein staining (score: van Bijsterveld score), GS grade Greenspan's grade, Scintigraphy technetium 99m-pertechnetate salivary gland scintigraphy, PG parotid gland, SMG submandibular gland, N number of patients with primary Sjögren's syndrome, NA not applicable

immune response is activated in the lesion of pSS, such as glandular and extra-glandular tissues. However, the platelet-related molecular cluster is associated with platelet activation, and its role in the pathophysiology of pSS remains unclear.

In this regard, Sarac et al. reported that patients with pSS with frequent episodic tension-type headache (FETH) had markedly decreased platelet serotonin levels (PSLs) and more common cerebral white matter signal hyperintensities (SHs) on brain magnetic resonance imaging than HCs. These findings appear to be associated with increased platelet serotonin release, indicating a more widespread cerebral vasculopathy in patients with pSS than in HCs $[29,30]$. Tomlins et al. developed a molecular concept model of prostate cancer progression using similar enrichment analysis and further confirmed molecular concepts that correlated with known histological features of prostate cancer progression [28]. The pSS-associated molecular concepts obtained by our method might therefore be useful at a clinical level. Recently, Delaleu et al. reported ontology-term network mapping of salivary gland fluid proteins examined using Human Discovery Multi-analyte Profile 1.0 (Myriad RBM, Austin, TX, USA) and identified immune (mainly B-cell-related) responses, $\mathrm{T}$ cell chemotaxis, and macrophage activation pathways [31]. This profile was confirmed by analysis of molecules in saliva using a similar enrichment analysis, and this analysis showed the association with molecular clusters involved in formation of glandular pathophysiology. That both studies identified partially similar molecular clusters is of interest. 
Various outcome measures used in previous clinical trials were based on glandular manifestations or symptoms, but not systemic manifestations. However, "activity indices" should contain both systemic and glandular features to evaluate the outcomes of new therapies. The ESSDAI was therefore developed as measure of disease activity in patients with systemic complications of pSS [18]. To date, ESSDAI is the only available disease activity index [32]. One of the strengths of our study includes the identification of biomarkers associated with the ESSDAI in patients with pSS, whose mean time of follow up was less than 5 years from diagnosis.

Our statistical extraction of surrogate biomarkers of the ESSDAI score also identified CXCL13, TNF-R2, CD48, BAFF, and PD-L2, which confirmed the findings of our validation study using a different cohort and methods. As these molecules are all involved in the immune response-related cluster, the immune response appears to be involved in this pathogenesis. These molecules might function as disease biomarkers for clinical follow up and as indicators of pSS pathogenesis. Very recently, CXCL13 was identified as a factor associated with the ESSDAI [33].

CXCL13 belongs to the CXC chemokine family. Follicular stromal cells, antigen-experienced $\mathrm{T}$ cells, and $\mathrm{T}$ helper (Th) follicular cells are all reported to produce CXCL13 [33-36], which recruits B cells to germinal centers. In patients with pSS, CXCL13 levels are upregulated in serum, saliva [37], and salivary gland tissue $[38,39]$. Based on our results, CXCL13 is associated with the pathogenesis of pSS, such as immunoglobulin production, and is linked to the activity of lymphadenopathy, glandular manifestation, interstitial lung disease (ILD) and biological status of the salivary glands.

TNF-R2, also known as p75 and TNFRSF1B, is mainly expressed in certain lymphocyte populations, such as regulatory $\mathrm{T}$ cells and $\mathrm{CD}^{+} \mathrm{T}$ cells, endothelial cells, microglia, oligodendrocytes, cardiac myocytes, thymocytes, and human mesenchymal stem cells [40]. It is reported that TNF-R2 also presents in a soluble form (sTNF-R2) and that plasma sTNF-R2 levels are increased in patients with active systemic lupus erythematosus and Behçet's disease [41-45]. In the examination of labial salivary gland tissues, Koski et al. [46] found that TNF$\alpha$, TNF-R1, and TNF-R2 were all expressed on vascular endothelial cells, ductal epithelial cells, and fibroblasts, but that only TNF-R1 was expressed on acinar end piece cells. TNF-R2 might therefore be associated with vascular or epithelial injury, which is a primary event in pSS.

CD48 is a member of the CD2 immunoglobulin superfamily, which includes SLAM proteins, and is expressed on the surface of lymphocytes and other immune cells, dendritic cells, and endothelial cells [47]. CD48 also exists in a soluble form (sCD48). Plasma sCD48 levels are elevated in patients with asthma, several infectious diseases including varicella, measles, and rubella, lymphoid leukemias, and arthritis [48-51]. However, the function of CD48 has not been clarified. Further investigation might reveal the association with the pathogenesis of pSS.

Several reports have been published on the role of BAFF in pSS. BAFF expression was increased in the salivary glands and the serum of patients with pSS [52]. Serum BAFF is particularly strongly upregulated in patients with pSS with lymphoproliferative disorders [15], and in patients with systemic lupus erthyematosus and rheumatoid arthritis [53-55]. Taken together with our present results, these previous findings suggest that BAFF might be associated with severe destruction of the salivary glands.

PD-L2 is a ligand of programmed cell death protein 1 (PD-1). It is reported that PD-L2 also has a soluble form [56]. Recent studies [56] have clarified significant roles of the PD-1/PD-L pathway in autoimmunity, including type 1 diabetes mellitus, systemic lupus erythematosus, rheumatoid arthritis and transplantation immunity, infectious immunity, and tumor immunity. PD-L2 might therefore modify PD-1/PD-L2 signaling and enhance immunoglobulin production, including autoantibodies, as PD-L2 expression has been observed on antigenpresenting cells (APCs) such as macrophages, dendritic cells, and activated T cells [57].

In addition, we extensively confirmed some characteristics of the molecules. To confirm whether these five proteins are specific for pSS, we compared serum concentration of them among four groups of patients (those with pSS and secondary Sjögren's syndrome (sSS), sicca syndrome and $\mathrm{HCs}$ ) as shown in Additional file 3: Figure $\mathrm{S} 2$. Increased serum levels of five proteins were observed in patients with pSS compared with $\mathrm{HCs}(P<0.05)$. Only CD48 levels were increased in patients with pSS compared with sicca syndrome patients, and there was no difference in the serum levels of four proteins was found in patients with pSS and patients with sicca syndrome. Increased serum levels of TNF-R2 and PD-L2 were observed in patients with sicca syndrome compared with HCs $(P<0.05)$. We consider that these age-matched and sex-matched patients with sicca syndrome, who did not satisfy any criteria for SS, include the patients who are clinically suspected to have a high probability of having SS. That may be one of the reasons why there is no significant difference between pSS and other sicca syndromes. We also analyzed the association between the five proteins and age, but there was no strong correlation (Additional file 4: Figure S3).

Several limitations to the present study warrant attention. First, the size of our study cohort is a little small for identifying serum biomarkers in systemic autoimmune diseases, which are heterogeneous diseases. Second, our 
study cohort did not include patients with long-term follow up, hampering the confirmation of changes in levels of candidate biomarkers depending on activity.

\section{Conclusions}

In conclusion, we comprehensively screened proteins related to disease activity and identified five clinically significant definitive serum biomarkers in patients with pSS. Further large-scale studies and analysis of the functional roles of these molecules are required to confirm their efficacy as markers for the evaluation of disease activity in pSS and the association with pathogenesis.

\section{Additional files}

Additional file 1: Table S1. Differentially expressed serum proteins in patients with pSS compared to HCs. (DOC $85 \mathrm{~kb}$ )

Additional file 2: Figure S1. Functional annotation of differentially expressed proteins in PSS patient sera. Nodes indicate molecular concepts or set of biologically related genes. Name of each node is indicated in black text on the node. The node size represents the proportion of differentially expressed gene symbols in the concepts (e.g., the "chemokine signaling pathway" and "extracellular region" concepts contain 14 and 58 genes, respectively). Length of lines between nodes represents degree of overlap between symbols. Colored lines indicate strength of functional relationship from strong to weak, as follows: red, yellow, green and gray. Green nodes indicate immune response-related molecular concepts, and red nodes indicate platelet-related molecular concepts. (TIF $9752 \mathrm{~kb}$ )

Additional file 3: Figure S2. Serum levels of five proteins in pSS, sSS, sicca syndrome and HCs. The five proteins were CXCL13, TNF-R2, CD48, BAFF and PD-L2. Primary SS (pSS), $n=58$; secondary SS (sSS), $n=6$; other sicca syndrome, $n=13$; healthy controls $(H C s), n=38$. Differences in quantitative variables were analyzed by the Mann-Whitney $U$ test when comparing two groups and by the Kruskal-Wallis test when comparing multiple groups. ${ }^{*} P$ value $<0.05$, which was considered significant. (TIF $33972 \mathrm{~kb}$ )

Additional file 4: Figure S3. Correlation between levels of five serum proteins and age in the validation cohort of patients with pSS and HCs. A Patients with $\mathrm{pSS}, n=58 ; \mathrm{B} \mathrm{HCs}, n=30$. Differences in quantitative variables were analyzed by the Pearson's correlation coefficient test $(P<0.05$ was considered significant). The correlation coefficients $(r)$ and $P$ values $(p)$ are shown. (TIF $33972 \mathrm{~kb}$ )

\section{Abbreviations}

Anti-SSA: anti-Sjögren's syndrome antigen A antibodies; anti-SSB: anti-Sjögren's syndrome antigen B antibodies; $\beta 2 M G$ : $\beta 2$-microglobulin; BAFF: B-cell activating factor; CXCL13: C-X-C motif chemokine 13; ELISA: enzyme-linked immunosorbent assay; EPHB-2: ephrin type-B receptor 2; ESSDAl: European League Against Rheumatism Sjögren's Syndrome Disease Activity Index; Fluorescein: fluorescein staining (score, van Bijsterveld score); GS grade: Greenspan's grade; IFN: interferon; Ig: immunoglobulin; LAG-3: lymphocyte activation gene-3; MXA: myxovirus resistance protein A; PD-L2: programmed cell death protein 1 ligand 2; PG: parotid gland; pSS: primary Sjögren's syndrome. Schirmer's test, Schirmer's-l test ( $\mathrm{mm} /$ $5 \mathrm{~min}$ ); RB test: Rose Bengal staining (score, van Bijsterveld score); Scintigraphy: technetium 99m-pertechnetate salivary gland scintigraphy; SLAMF2: CD48 signaling lymphocytic activation molecule 2; SMG: submandibular gland; TNF: tumor necrosis factor; TNF-R2: tumor necrosis factor receptor 2.

\section{Competing interests}

$\mathrm{AN}, \mathrm{KYO}, \mathrm{HY}, \mathrm{KYa}$, RM, and $\mathrm{AY}$ have no competing interests. YK, YG, MT, and TM are employees of Takeda Pharmaceutical Co., Ltd. KS has received research grants from Eisai Co., Ltd., and Bristol-Myers Squibb. TT has received grants from Astellas Pharma; Bristol-Myers K.K.; Chugai Pharmaceutical Co. Ltd.; Daiichi Sankyo Co., Ltd.; Eisai Co., Ltd.; Mitsubishi Tanabe Pharma Co.; Pfizer Japan Inc.; Santen Pharmaceutical Co., Ltd.; Takeda Pharmaceutical Co.,
Ltd.; Teijin Pharma Ltd.; AbbVie GK.; Asahikasei Pharma Corp.; Taisho Toyama Pharmaceutical Co., Ltd.; and SymBio Pharmaceuticals Ltd.; speaking fees from AbbVie GK.; Bristol-Myers K.K.; Chugai Pharmaceutical Co., Ltd.; Eisai Co., Ltd.; Janssen Pharmaceutical K.K.; Mitsubishi Tanabe Pharma Co.; Pfizer Japan Inc.; and Takeda Pharmaceutical Co., Ltd.; Astellas Pharma; and Diaichi Sankyo Co., Ltd.; Celtrion; and Nipponkayaku Co., Ltd.; and consultant fees from Astra Zeneca K.K.; Eli Lilly Japan K.K.; Novartis Pharma K.K.; Mitsubishi Tanabe Pharma Co.; and Asahi Kasei Medical K.K.; AbbVie GK.; Daiichi Sankyo Co., Ltd.; Bristol-Myers K.K.; and Nipponkayaku Co., Ltd.

\section{Authors' contributions}

$\mathrm{AN}, \mathrm{KS}$, and TT were involved in drafting the manuscript. All authors were involved in revising it critically for important intellectual content, and revising the final version. $T$ had full access to all data in the study and takes responsibility for the integrity of the data and the accuracy of the data analysis. $A N, K S, Y K, T M, A Y$, and TT participated in the study conception and design. AN, $\mathrm{KS}, \mathrm{YK}, \mathrm{YG}, \mathrm{MT}, \mathrm{KYO}, \mathrm{HY}, \mathrm{Kya}$, and RM participated in the acquisition of data, and analysis and interpretation of data. All authors read and approved the manuscript.

\section{Acknowledgements}

The authors thank the patients and staff. The authors also thank DMC Corp. for editing this manuscript. This study was funded by a collaborative research grant from Takeda Pharmaceutical Co., Ltd., and internal research grants from Keio University.

\section{Author details}

'Division of Rheumatology, Department of Internal Medicine, Keio University School of Medicine, 35 Shinanomachi, Shinjuku-ku, Tokyo 160-8582, Japan. ${ }^{2}$ Inflammation Drug Discovery Unit, Pharmaceutical Research Division, Takeda Pharmaceutical Company Limited, 2-26-1 Muraokahigashi, Fujisawa city, Kanagawa 251-0012, Japan. ${ }^{3}$ Department of Microbiology and Immunology, Keio University School of Medicine, 35 Shinanomachi, Shinjuku-ku, Tokyo 160-8582, Japan.

Received: 4 January 2016 Accepted: 28 April 2016

Published online: 14 May 2016

\section{References}

1. Mariette $X$, Seror R, Quartuccio $L$, et al. Efficacy and safety of belimumab in primary Sjögren's syndrome: results of the BELISS open-label phase II study. Ann Rheum Dis. 2015;74:526-31.

2. Brkic Z, Versnel MA. Type I IFN signature in primary Sjögren's syndrome patients. Expert Rev Clin Immunol. 2014;10:457-67.

3. Mavragani CP, Moutsopoulos HM. Sjögren's syndrome. Annu Rev Pathol Mech Dis. 2014;9:273-85.

4. Devauchelle-Pensec V, Mariette X, Jousse-Joulin S, et al. Treatment of primary Sjögren syndrome with rituximab: a randomized trial. Ann Intern Med. 2014;160:233-42

5. Guellec D, Cornec D, Jousse-Joulin S, et al. Diagnostic value of labial minor salivary gland biopsy for Sjögren's syndrome: a systematic review. Autoimmunity Reviews. 2013;12:416-20.

6. Zhou D, McNamara NA. Macrophages: important players in primary Sjögren's syndrome? Expert Rev Clin Immunol. 2014;10:513-20.

7. Al-Tarawneh SK, Border MB, Dibble CF, et al. Defining salivary biomarkers using mass spectrometry-based proteomics: a systematic review. OMICS. 2011;15:353-61.

8. Deutsch $\mathrm{O}$, Krief $\mathrm{G}$, Konttinen $\mathrm{YT}$, et al. Identification of Sjögren's syndrome oral fluid biomarker candidates following high-abundance protein depletion. Rheumatology (Oxford). 2015;54:884-90.

9. Fleissig Y, Deutsch O, Reichenberg E, et al. Different proteomic protein patterns in saliva of Sjögren's syndrome patients. Oral Dis. 2009;15:61-8.

10. Giusti L, Baldini C, Bazzichi L, et al. Proteomic diagnosis of Sjögren's syndrome. Expert Rev Proteomics. 2007;4:757-67.

11. Hu S, Vissink A, Arellano M, et al. Identification of autoantibody biomarkers for primary Sjögren's syndrome using protein microarrays. Proteomics. 2011; 11:1499-507.

12. Peluso G, De Santis M, Inzitari R, et al. Proteomic study of salivary peptides and proteins in patients with Sjögren's syndrome before and after pilocarpine treatment. Arthritis Rheum. 2007;56:2216-22. 
13. Ryu OH, Atkinson JC, Hoehn GT, et al. Identification of parotid salivary biomarkers in Sjögren's syndrome by surface-enhanced laser desorption/ ionization time-of-flight mass spectrometry and two-dimensional difference gel electrophoresis. Rheumatology. 2006;45:1077-86.

14. Baldini C, Giusti L, Ciregia F, et al. Proteomic analysis of saliva: a unique tool to distinguish primary Sjögren's syndrome from secondary Sjögren's syndrome and other sicca syndromes. Arthritis Res Ther. 2011;13(6):R194.

15. Quartuccio L, Salvin S, Fabris M, et al. BLyS upregulation in Sjögren's syndrome associated with lymphoproliferative disorders, higher ESSDAI score and B-cell clonal expansion in the salivary glands. Rheumatology (Oxford). 2013;52:276-81.

16. Gottenberg JE, Seror R, Miceli-Richardo C, et al. Serum levels of beta2microglobulin and free light chains of immunoglobulins are associated with systemic disease acitivity in primary Sjögren's syndrome. data at enrollment in the prospective ASSESS cohort. PLoS One. 2013;8(5):e59868.

17. Maria NI, Brkic Z, Waris M, et al. MxA as a clinically applicable biomarker for identifying systemic interferon type I in primary Sjögren's syndrome. Ann Rheum Dis. 2014;73:1052-9.

18. Seror R, Ravaud P, Bowman S, et al. EULAR Sjögren's syndrome disease activity index: development of a consensus systemic disease activity index for primary Sjögren's syndrome. Ann Rheum Dis. 2010;69:1103-9.

19. Moerman RV, Arends S, Meiners PM, et al. EULAR Sjögren's Syndrome Disease Activity Index (ESSDAI) is sensitive to show efficacy of rituximab treatment in a randomized controlled trial. Ann Rheum Dis. 2014;73:472-4.

20. Vitali C, Bombardieri S, Jonsson R, et al. Classification criteria for Sjögren's syndrome: a revised version of the European criteria proposed by the American-European Consensus Group. Ann Rheum Dis. 2002;6:554-8.

21. Shiboski SC, Shiboski CH, Criswell L, et al. American College of Rheumatology classification criteria for Sjögren's syndrome: a data-driven, expert consensus approach in the Sjögren's International Collaborative Clinical Alliance cohort. Arthritis Care Res. 2012;64:475-87.

22. Tsuboi $\mathrm{H}$, Hagiwara $\mathrm{S}$, Asashima $\mathrm{H}$, et al. Validation of different sets of criteria for the diagnosis of Sjögren's syndrome in Japanese patients. Mod Rheumatol. 2013;23:219-25.

23. Brito-Zerón P, Kostov B, Solans R, et al. Systemic activity and mortality in primary Sjögren syndrome: predicting survival using the EULAR-SS Disease Activity Index (ESSDAI) in 1045 patients. Ann Rheum Dis. 2014;0:1-8. doi:10. 1136/annrheumdis-2014-206418.

24. Rohloff JC, Gelinas AD, Jarvis TC, et al. Nucleic acid ligands with protein-like side chains: modified aptamers and their use as diagnostic and therapeutic agents. Mol Ther Nucleic Acids. 2014;3:e201.

25. Gold L, Ayers D, Bertino J, et al. Aptamer-based multiplexed proteomic technology for biomarker discovery. PLoS One. 2010;5(12):e15004.

26. Webber J, Stone TC, Katilius E, et al. Proteomics analysis of cancer exosomes using a novel modified aptamer-based array (SOMAscan ${ }^{\mathrm{TM}}$ ) platform. Mol Cell Proteomics. 2014;13:1050-64.

27. Yoshimoto K, Tanaka M, Kojima M, et al. Regulatory mechanisms for the production of BAFF and IL-6 are impaired in monocytes of patients of primary Sjögren's syndrome. Arthritis Res Ther. 2011;13(5):R170.

28. Tomlins SA, Mehra R, Rhodes DR, et al. Integrative molecular concept modeling of prostate cancer progression. Nat Genet. 2007;39:41-51.

29. Markeljevic J, Sarac H, Bozina N, et al. Serotonin transporter gene polymorphisms: relation with platelet serotonin level in patients with primary Sjogren's syndrome. J Neuroimmunol. 2015;282:104-9.

30. Sarac H, Markeljevic J, Erdeljic V, et al. Signal hyperintensities on brain magnetic resonance imaging in patients with primary Sjögren's syndrome and frequent episodic tension-type headache; relation to platelet serotonin level and disease activity. J Rheumatol. 2013;40:1360-6.

31. Delaleu N, Mydel P, Kwee I, et al. High fidelity between saliva proteomics and the salivary glands' biological state defines biomarker-signatures for primary Sjögren's syndrome. Arthritis Rheumatol. 2015;67:1084-95.

32. Campar A, Isenberg DA. Primary Sjögren's syndrome activity and damage indices comparison. Eur J Clin Invest. 2010;40:636-44

33. Nocturne G, Seror R, Fogel O, et al. CXCL13 and CCL11 serum levels and lymphoma and disease activity in primary Sjögren Syndrome. Arthritis Rheumatol. 2015. doi:10. 1002/art.393915.

34. Manzo A, Vitolo B, Humby F, et al. Mature antigen-experienced T helper cells synthesize and secrete the B cell chemoattractant CXCL13 in the inflammatory environment of the rheumatoid joint. Arthritis Rheum. 2008; 58:3377-87.
35. Gunn MD, Ngo VN, Ansel KM, et al. A B-cell-homing chemokine made in Iymphoid follicles activates Burkitt's lymphoma receptor-1. Nature. 1998;391: 799-803.

36. Cyster JG, Ansel KM, Reif K, et al. Follicular stromal cells and lymphocyte homing to follicles. Immunol Rev. 2000;176:181-93.

37. Kramer JM, Klimatcheva E, Rothstein TL, et al. CXCL13 is elevated in Sjögren's syndrome in mice and humans and is implicated in disease pathogenesis. J Leukoc Biol. 2013;94:1079-89.

38. Barone F, Bombardieri M, Manzo A, et al. Association of CXCL13 and CCL21 expression with the progressive organization of lymphoid-like structures in Sjögren's syndrome. Arthritis Rheum. 2005:52:1773-84.

39. Barone F, Bombardieri M, Rosado MM, et al. CXCL13, CCL21, and CXCL12 expression in salivary glands of patients with Sjögren's syndrome and MALT lymphoma: association with reactive and malignant areas of lymphoid organization. J Immunol. 2008;180:5130-40.

40. Fraustman DL, Davis M. TNF receptor 2 and disease: autoimmunity and regenerative medicine. Front Immunol. 2013;4:478.

41. Patel M, Oni L, Midgley A, et al. Increased concentration of plasma TNFR1 and TNFR2 in paediatric lupus nephritis. Lupus. 2016;0:1-5. doi:10.1177/ 0961203316631634

42. Horiuchi T, Mitoma H, Harashima S, et al. Transmembrane TNF-a: structure, function and interaction with anti-TNF agents. Rheumatology. 2010;49: 1215-28.

43. Svenungsson E, Gunnarsson I, Fei GZ, et al. Elevated triglycerides and low levels of high-density lipoprotein as markers of disease activity in association with up-regulation of the tumor necrosis factor alpha/tumor necrosis factor receptor system in systemic lupus erythematosus. Arthritis Rheum. 2003;48: 2533-40.

44. Ferreira GA, Teixeira A, Calderano DC, et al. Atorvastatin reduced soluble receptors of TNF-alpha in systemic lupus erythematosus. Clin Exp Rheumatol. 2016;34:42-8.

45. Turan B, Pfister K, Diener PA, et al. Soluble tumour necrosis factor receptors sTNFR1 and sTNFR2 are produced at sites of inflammation and are markers of arthritis activity in Behçet's disease. Scand J Rheumatol. 2008;37:135-41.

46. Koski H, Janin A, Humphreys-Beher MG, et al. Tumor necrosis factor-alpha and receptor for it in labial salivary glands in Sjögren's syndrome. Clin Exp Rheumatol. 2001:19:131-7.

47. Elishmereni M, Levi-Schaffer F. CD48: a co-stimulatory receptor of immunity. Int J Biochem Cell Biol. 2011;43:25-8.

48. Gangwar RA et al. SCD48 is anti-inflammatory in S. aureus Enterotoxin B induced eosinophilic inflammation. Allergy. 2016. doi:10.1111/all.12851.

49. Ariel $\mathrm{M}$ et al. CD48 is an allergen and IL-3-induced activation molecule on eosinophils. J Immunol. 2006;177:77-83.

50. Smith GM et al. Detection of a soluble form of the leukocyte surface antigen CD48 in plasma and its elevation in patients with lymphoid leukemias and arthritis. J Clinical Immunol. 1997;17:502-9.

51. Boles KS et al. 2B4 (CD244) and CS1: novel members of the CD2 subset of the immunoglobulin superfamily molecules expressed on natural killer cells and other leukocytes. Immunol Rev. 2001;181:234-49.

52. Candon S, Gottenberg JE, Bengoufa D, et al. Quantitative assessment of antibodies to ribonucleoproteins in primary Sjögren's syndrome: correlation with B-cell biomarkers and disease activity. Ann Rheum Dis. 2009;68:1208-12.

53. Mariette X, Roux S, Zhang J, et al. The level of BLyS (BAFF) correlates with the titre of autoantibodies in human Sjögren's syndrome. Ann Rheum Dis. 2003:62:168-71.

54. Zhang J, Roschke V, Baker KP, et al. Cutting edge: a role for B lymphocytestimulator in systemic lupus erythematosus. J Immunol. 2001;166:6-10.

55. Cheema GS, Roschke V, Hilbert DM, et al. Elevated serum B lymphocyte stimulator levels in patients with systemic immune-based rheumatic diseases. Arthritis Rheum. 2001:44:1313-9.

56. Dai $S$, Jia R, Zhang $X$, et al. The PD-1/PD-Ls pathway and autoimmune diseases. Cell Immunol. 2014;290:72-9.

57. Messal N, Serriari NE, Pastor S, et al. PD-L2 is expressed on activated human T cells and regulates their function. Mol Immunol. 2011;48:2214-9. 